\title{
Erectile Dysfunction in Men With Psoriatic Arthritis: A Population-based Cohort Study
}

\author{
Katelynn M. Wilton ${ }^{1}$ (D), Sara J. Achenbach ${ }^{2}$, Paras Karmacharya ${ }^{3}$ (D) Floranne C. Ernste $^{3}(\mathbb{D}$, \\ Eric L. Matteson ${ }^{4}$, and Cynthia S. Crowson ${ }^{5}$ (iD)
}

\begin{abstract}
Objective. To define the incidence of erectile dysfunction (ED) in a population-based cohort of men with psoriatic arthritis (PsA).

Methods. Data pertaining to demographics, ED, and potential confounding diagnosis were extracted from a comprehensive medical record system for a population-based cohort of men with PsA and an age-matched male comparator cohort. Cumulative incidence of ED adjusted for competing risk of death was compared between the 2 cohorts.

Results. There were 128 age-matched pairs of men with PsA and without PsA in the described cohorts. At baseline, there was a $7 \%$ prevalence of ED in men with PsA prior to diagnosis compared to a 3\% prevalence of ED in the comparator cohort $(P=0.16)$. After PsA diagnosis/index date, diagnosis with PsA was associated with an increased risk of ED (age-adjusted HR 1.45, 95\% CI 0.79-2.68), but this association did not reach statistical significance. This was based on 24 cases of ED in the men with PsA and 18 cases within the comparator cohort. No confounding factors or ED treatment strategies differed significantly between men with PsA and ED and comparators with ED.

Conclusion. Men with PsA may have an increased risk of ED, which was detected but likely underpowered in this study. Whether this difference is secondary to higher prevalence of traditional risk factors of ED in men with PsA compared to the general population will require further study.
\end{abstract}

Key Indexing Terms: epidemiology, erectile dysfunction, psoriatic arthritis, sexual dysfunction, sexual health

Psoriatic arthritis (PsA) is a systemic inflammatory disease which, in addition to chronic inflammatory joint and entheseal symptoms, confers an increased risk of comorbidities, including both

KMW was supported by the National Institutes of General Medical Sciences (grant T32-GM-65841) and is supported by the Mayo Clinic College of Medicine's Medical Scientist Training Program. Research reported in this publication was supported by the National Institute of Aging of the National Institutes of Health (NIH) under award number R01 AG034676 and grant number UL1TR002377 from the National Center for Advancing Translational Sciences (NCATS), one of the institutes of the NIH. The content is solely the responsibility of the authors and does not necessarily represent the official views of the NIH.

${ }^{1}$ K.M. Wilton, BS, Medical Scientist Training Program, Mayo Clinic College of Medicine and Science; ${ }^{2}$ S.J. Achenbach, MS, Division of Biomedical Statistics and Informatics, Mayo Clinic College of Medicine and Science; ${ }^{3}$ P. Karmacharya, MBBS, F.C. Ernste, MD, Division of Rheumatology, Mayo Clinic College of Medicine and Science; ${ }^{4}$ E.L. Matteson, MD, MPH, Division of Rheumatology, and Division of Epidemiology, Department of Health Sciences Research, Mayo Clinic College of Medicine and Science; ${ }^{5}$ C.S. Crowson, PhD, Division of Biomedical Statistics and Informatics, and Division of Rheumatology, Mayo Clinic College of Medicine and Science, Rochester, Minnesota, USA.

The authors declare no conflicts of interest.

Address correspondence to Dr. C.S. Crowson, Mayo Clinic, 200 1st St. SW, Rochester, MN 55905, USA.Email: Crowson@mayo.edu.

Full Release Article. For details see Reprints and Permissions at jrheum.org. Accepted for publication October 2, 2020. cardiovascular disease (CVD) ${ }^{1-8}$ and sexual dysfunction ${ }^{9,10,11,12}$. PsA is associated with increased mortality, with CVD as the leading cause of death ${ }^{13}$. Cardiovascular (CV) events and symptoms are more common in patients with PsA compared to the general population ${ }^{1,14}$. Although the majority of $\mathrm{CV}$ risk has been attributed to traditional CV risk factors, whether psoriatic disease activity plays a role in $\mathrm{CV}$ risk is controversial, with some studies supporting a role for psoriatic disease activity in $\mathrm{CV}$ risk ${ }^{1,14,15,16}$, while others do not ${ }^{17}$. This is complicated by the increased prevalence of CV risk factors in patients with $\mathrm{PsA}^{18}$.

Sexual and erectile dysfunction (ED) are common in the general population, and are associated with increased CV risk. Specifically, sexual dysfunction is a broad term that encompasses many forms of sexual conditions, including areas of desire, arousal, orgasm, and pain during sexual intercourse ${ }^{19}$. These can include ED, low sexual desire, vagismus, dyspareunia, inability to orgasm, marital difficulties, and psychogenic aversion to sexual activity. Sexual dysfunction can apply to either male or female patients, and can be the result of a variety of medical or psychological states ${ }^{20}$. The heterogeneity of sexual dysfunction makes it a difficult area to study, especially in relation to epidemiologic risk. $\mathrm{ED}$, in contrast, is a specific diagnosis that reflects the decreased ability of a man to have and maintain an erection adequate for his desired sexual activity ${ }^{21,22}$. Although more precise definitions of ED have been made in a research context, such as decreased penile blood flow by Doppler ultrasound, these are rarely used 
in clinical practice and are therefore not generally useful in an epidemiologic context $\mathrm{t}^{23,24,25,26}$. ED is a more specific diagnosis, but can still be secondary to both medical and psychogenic causes. From a vascular perspective, ED can be secondary to atherosclerotic disease, which limits blood flow to the penile vasculature and prevents adequate erection ${ }^{27,28,29}$. Neurologically, if there is compromise at any level of the nervous system leading to the innervation of the penis, this can also result in $\mathrm{ED}^{30,31}$. From a psychological perspective, if a patient is experiencing stress, depression, marital complications, or other psychologic states, he may not experience an erection upon initiating sexual activity but will still experience nocturnal erections, which are generally unaffected by the psychological state ${ }^{32}$.

Vascular ED has even been labeled as a "harbinger of CVD," generally occurring $2-5$ years before $\mathrm{CV}$ events or symptoms ${ }^{33,34}$. Given this close association between CVD and vascular ED, men with PsA may have an increased rate of ED, potentially occurring earlier or more commonly than in the general population. Moreover, patients with PsA have a higher risk of psychiatric comorbidities such as anxiety and depression, and the psychological effects of psoriasis and PsA may be associated with sexual dysfunction ${ }^{35}$. Previous studies have shown that $\mathrm{Ps}^{9}{ }^{9,10,12}$ and psoriatic disease activity ${ }^{36,37,38}$ are both risk factors for ED in men with psoriasis, a population known to have an increased risk of sexual dysfunction. However, unlike sexual dysfunction, and despite this implied association, the incidence of ED in men with PsA has not been previously analyzed in a population-based cohort and has been analyzed minimally by other methods ${ }^{39}$. By determining the incidence of ED in men with PsA, we can begin to explore the relationship between vascular ED and $\mathrm{CV}$ risk within this population of men with a heightened risk of $\mathrm{CV}$ morbidity and mortality.

The specific diagnostic nature of ED [encompassed by the International Classification of Diseases, 10th revision (ICD-10) codes], its close association with CV risk, as well as the clear link between vascular ED and CV risk, suggested that ascertaining the incidence of $\mathrm{ED}$, with a focus on vascular etiologies, may help to further delineate CV risk in PsA and inform risk estimates for $\mathrm{CV}$ risk in men with PsA. Given the association of $\mathrm{CV}$ and psychiatric diseases with $\mathrm{ED}$, as well as previous studies suggesting an association between ED and psoriatic disease, we aimed to determine the incidence of ED in a population-based cohort of men with PsA in relation to age-matched male comparators.

\section{MATERIALS AND METHODS}

Studypopulations. The Rochester Epidemiology project ${ }^{40}$ was used to identify PsA and comparator cohorts, who were composed of residents of Olmsted County (Minnesota, USA) aged 18 years or older, as previously published ${ }^{41}$. The incident PsA cohort included men diagnosed with PsA [according to the ClASsification for Psoriatic ARthritis criteria (CASPAR) ${ }^{42}$ ] in Olmsted County between January 1, 1970, and December 31, 2008. A comparator cohort of men of similar age without PsA was established to match the PsA cohort. Longitudinal follow-up was completed until death, migration from Olmsted county, or December 31, 2018. Patients who declined access to their medical records for research purposes were excluded as per Minnesota law. However, no patients were excluded for this reason in our study, as noted in Figure 1. This study was approved by the institutional review boards at the Mayo Clinic (17-002943) and the Olmsted Medical Center (024-OMC-17).

Data abstraction. Relevant data were abstracted from the medical record and used in conjunction with previously extracted data ${ }^{1,41}$. New data collection included information about ED diagnosis (date of diagnosis, type of ED), risk factors for ED (alcohol concerns, hypogonadism, thyroid disease, Peyronie disease, penile or spinal trauma, systemic neurologic disease, radical prostatectomy, or pelvic radiation), treatments for ED [phosphodiesterase 5 (PDE5) inhibitors, testosterone, penile self-injection, penile vacuum device use, or surgical penile device placement], and common underlying medications (antihypertensives, antidepressants, antiandrogens) that might predispose to ED. Specifically, ED diagnoses was extracted after identifying relevant ICD-9 and ICD-10 codes. KMW then reviewed the medical records and ensured that the diagnosis was present and made by a physician or midlevel provider. The medical specialty (urology, internal medicine, family medicine, other) of the provider was also recorded. The diagnosis was made in all cases per usual clinical decision-making used at the institutions. No formal ED diagnostic criteria were required. In cases where the clinician formally considered and described in the medical record psychological components that could be primarily responsible for or contributing to the $\mathrm{ED}^{32}$, "possible psychogenic ED" was noted. These data were recorded in the secure Research Electronic Data Capture (REDCap) system without personally identifying information.

Statistical methods. Descriptive statistics (number, percentage, mean, SD) were used to summarize the data. For continuous numeric data, characteristics of the cohorts were compared by Kruskal-Wallis test for statistical significance. For categorical groups, chi-square testing was used to determine statistically significant differences. Cumulative incidence of ED in each of the cohorts was estimated, adjusting for competing risk of death ${ }^{43}$, which accounts for those who died before experiencing ED without censoring, and thus avoids overestimating the incidence of ED in surviving members of the cohort. Subjects who were diagnosed with ED before their respective diagnosis date/index date were excluded from the cumulative incidence analysis. Analyses were performed using SAS version 9.4 (SAS Institute) and R 3.6.2 ( $\mathrm{R}$ foundation for statistical computing).

\section{RESULTS}

There were 128 matched pairs of male PsA patients and age-matched male comparators with similar ages (PsA mean 42.3, SD 13.1; comparator mean 42.4, SD 13.1, $P=0.97$ ). There was no difference in the baseline ED prevalence with $7 \%$ of the men with PsA $(9 / 128)$ and $3 \%$ of the comparators $(4 / 128, P=0.16)$ having an ED diagnosis preceding their PsA diagnosis/index date (Figure 1). The mean follow-up was 18.8 years (SD 10.5) in the PsA cohort and 19.1 years (SD 9.4) in the comparator cohort.

During the follow-up period, 24 of 119 men with PsA were diagnosed with ED after their PsA diagnosis (20-yr cumulative incidence 19\%, 95\% CI 13-29\%). In the comparator cohort, 18 of 124 men were diagnosed with ED after their index date (20-yr cumulative incidence $13 \%, 95 \%$ CI 8-21\%). As shown in Figure 2, the cumulative incidence of ED in PsA was higher than the cumulative incidence in comparators, with an increasing divide between the men with PsA and the comparators with time. However, the $45 \%$ increased risk of ED in men with PsA compared to men without PsA did not reach statistical significance (age-adjusted HR 1.45, 95\% CI 0.79-2.68). Of note, the specialty of the diagnosing clinicians was varied in both groups and included urologists, internal medicine, and family medicine 

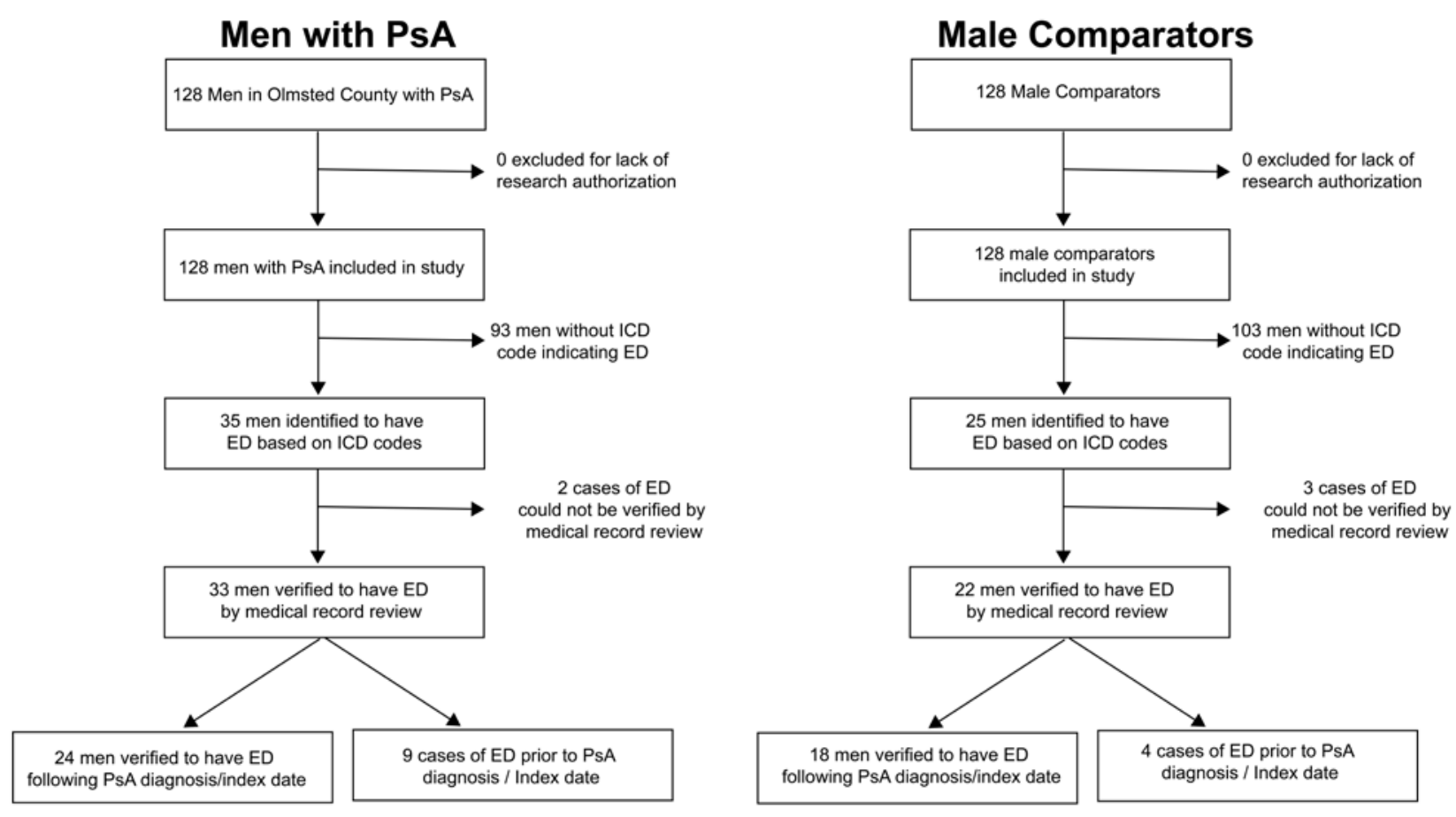

Figure 1. Study design and exclusions. The number of initial men with PsA (left column), and male comparators (right column) are shown. There were no exclusions in this study due to lack of consent. A total of 13 study subjects were excluded for having a diagnosis of ED before their diagnosis or index date. ED: erectile dysfunction; ICD: International Classification of Diseases; PsA: psoriatic arthritis.

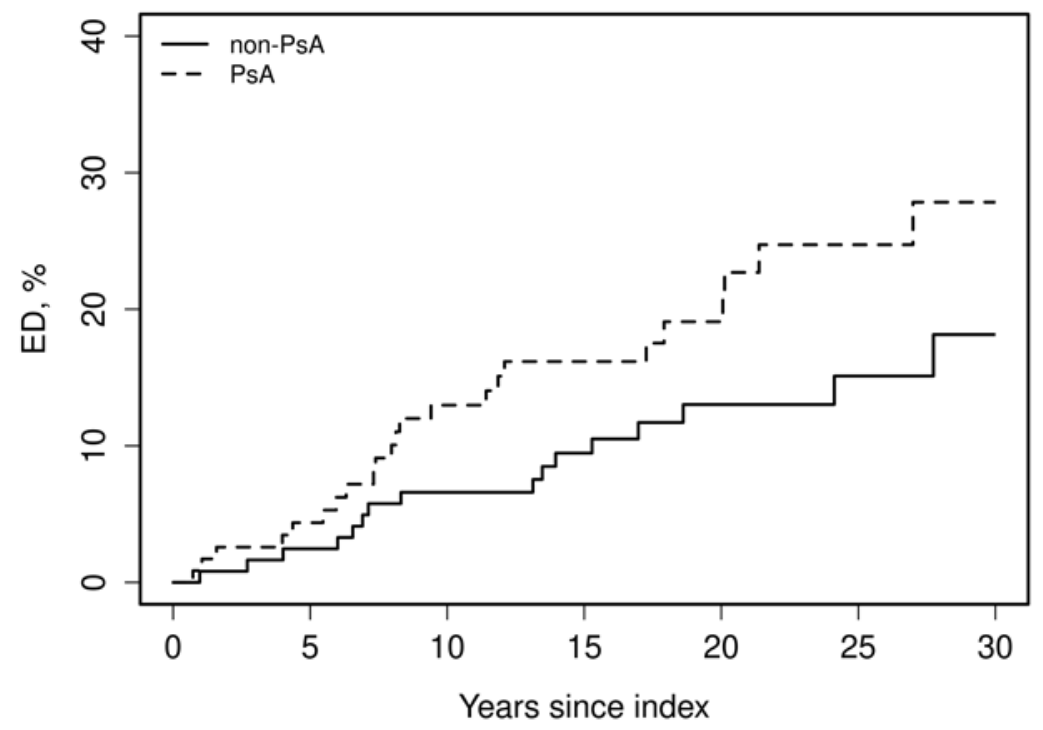

Figure 2. Cumulative incidence of ED in men with PsA. The cumulative incidence of ED was assessed over time after initial PsA diagnosis or respective index date in the comparator cohort. The incidence of ED was adjusted for competing risk of death, and those who developed ED before the index date were excluded. Analysis was adjusted for age. There were 18 cases of ED in the comparator cohort and 24 in the PsA cohort (HR 1.45, 95\% CI 0.79-2.68). ED: erectile dysfunction; PsA: psoriatic arthritis.

clinicians. There was no statistically significant difference in the specialty of the clinicians that diagnosed $\mathrm{ED}$ between the 2 groups $(P=0.55)$.

The men who developed ED in the PsA and comparator cohorts were further analyzed to determine if there were any clear confounding factors. As shown in Table 1, the potential confounding conditions, including psychological factors, medication use, relevant trauma, cancer therapies, and other 
Table 1. Potential diagnostic confounders and initial treatment strategies in men with PsA and ED.

\begin{tabular}{|c|c|c|c|}
\hline & $\begin{array}{c}\text { PsA, } \\
\mathrm{n}=24\end{array}$ & $\begin{array}{c}\text { Non-PsA, } \\
\mathrm{n}=18\end{array}$ & $P$ \\
\hline \multicolumn{3}{|l|}{ Specialty of diagnosing clinician } & 0.55 \\
\hline Urology & 7 (29) & $3(17)$ & \\
\hline Internal medicine & $8(33)$ & $9(50)$ & \\
\hline Family medicine & $8(33)$ & $6(33)$ & \\
\hline Other subspecialist & $1(4)$ & $0(0)$ & \\
\hline \multicolumn{4}{|l|}{ Potential confounders } \\
\hline BMI, kg/m², mean (SD) & $30.3(4.9)$ & $28.9(2.2)$ & 0.49 \\
\hline Possible psychogenic ED & $3(13)$ & $4(22)$ & 0.40 \\
\hline Alcohol concerns & $7(29)$ & $5(28)$ & 0.92 \\
\hline Antihypertensive use & $11(46)$ & $8(44)$ & 0.93 \\
\hline Antidepressant use & $5(21)$ & $3(17)$ & 0.73 \\
\hline Antiandrogen use & $0(0)$ & $0(0)$ & - \\
\hline Hypogonadism diagnosis & $0(0)$ & $0(0)$ & - \\
\hline Thyroid disease diagnosis & $0(0)$ & $0(0)$ & - \\
\hline Peyronie disease & $1(4)$ & $1(6)$ & 0.83 \\
\hline Penile trauma & $0(0)$ & $1(6)$ & 0.24 \\
\hline Spinal cord trauma & $0(0)$ & $0(0)$ & - \\
\hline Neurological disease & $0(0)$ & $1(6)$ & 0.24 \\
\hline Radical prostatectomy & $2(8)$ & $0(0)$ & 0.21 \\
\hline Radiation therapy & $0(0)$ & $0(0)$ & - \\
\hline \multicolumn{4}{|l|}{ Treatments in the first year } \\
\hline PDE5 inhibitor & $16(67)$ & $15(83)$ & 0.22 \\
\hline Testosterone replacement & $0(0)$ & $0(0)$ & - \\
\hline Penile self-injection & $0(0)$ & $0(0)$ & - \\
\hline Penile vacuum device & $0(0)$ & $0(0)$ & - \\
\hline Penile device placement & $1(4)$ & $0(0)$ & 0.38 \\
\hline
\end{tabular}

Values are n (\%) unless otherwise specified. ED: erectile dysfunction; PDE5: phosphodiesterase 5; PsA: psoriatic arthritis.

predisposing diagnoses, were compared between the 2 groups. In addition, BMI was not statistically different between the 2 groups $(P=0.49)$. There were no significant differences in investigated confounding factors between the cohorts, which may have been due to the small number of affected individuals.

Additionally, the initial treatment strategies (started within the first year after ED diagnosis) were similar between the 2 cohorts. As shown in Table 1, PDE5 inhibitors were highly used in both cohorts, being prescribed within the first year after diagnosis in $67 \%$ of men with PsA and ED, and $83 \%$ of comparator men with $\mathrm{ED}(P=0.22)$. Testosterone replacement, penile self-injection, and vacuum device were not used within the first year after ED diagnosis in this study.

\section{DISCUSSION}

In this study, PsA was associated with a trend toward an increased risk of ED. There were no clear confounding factors observed in patients with ED.

To our knowledge, this is the first population-based study to directly investigate the incidence of ED in men with PsA. Previous studies found an increased risk of ED in patients with psoriasis who also had $\mathrm{PsA}^{9,10,12}$. The findings of this study are consistent with previous studies, although the increased risk in this study did not reach statistical significance due to the low numbers of ED cases ( 24 and 18 cases in the PsA and comparator cohorts, respectively). Of note, the incidence of $\mathrm{ED}$ in this study was relatively low within the comparator cohort. However, this was consistent with prior reports of ED incidence and prevalence in men residing in Olmsted County of equivalent age ${ }^{44,45}$.

When considering the implications of ED diagnosis in men with PsA, there are several obvious areas of consideration. First, it has been previously shown that psoriasis has been linked to psychological sequelae that cause sexual dysfunction ${ }^{46,47}$. This is particularly prominent in men who are affected with genital psoriasis lesions ${ }^{46}$. However, even in men without psoriasis, anxiety, depression, and marital complications can lead to $\mathrm{ED}^{32}$. Although we did not directly assess the predominance of psychologic comorbidities in this study, we did find that clinicians were considering psychogenic ED in both men with PsA and male comparators (Table 1) at similar rates. There was also similar antidepressant use in both groups. Although these measures are only proxies of underlying psychological disorders, they do indicate that a large percentage of the ED seen in men with PsA is not fully accounted for by psychological pathologies. The underlying psychological effects of PsA and their implications for sexual dysfunction are an underexplored area that could greatly increase quality of life for men with PsA. Consideration of sexual comorbidities in men with PsA is important both for future research directions and for the care of individuals with PsA. It will be important for clinicians to initiate these conversations because early recognized ED may help to highlight underlying psychological needs.

A secondary area in which diagnosis of ED may have important implications is in the area of CV morbidity and mortality. Given the common underlying pathology involving atherosclerotic lesions, lipid deposition, and arterial insufficiency, vascular ED is physiologically linked to $\mathrm{CVD}^{33,34}$. In the general population, the implications of $\mathrm{ED}$ on future $\mathrm{CV}$ health have been clear: ED generally precedes CV events and symptoms ${ }^{33,34}$. However, in men with PsA, the implication of ED is less clear. Based on current knowledge, the prevailing assumption would be that ED also signals increased risk in men with PsA and should prompt consideration of $\mathrm{CV}$ risk; however, at present, this remains an assumption. Future work to detail the link between ED and CV health in men with PsA, while considering their baseline heightened CV risk, will help to guide more directed interventions and hopefully inform treatment of a major cause of morbidity and mortality in PsA.

This study has several strengths and limitations inherent to its design. First, to our knowledge, this was the first population-based cohort to investigate the incidence of ED in men with PsA. Given the retrospective population-based design and comprehensive record system, this study was able to account for all men diagnosed with ED by a physician, and able to be considered in the context of a comprehensive medical record. However, it was limited in its ability to detect subclinical or undiagnosed ED. This study focused on ED, a more specific diagnosis than that of previous studies investigating overall sexual dysfunction, which may be confounded by sexual aversion secondary to genital 
psoriatic disease and joint pain ${ }^{11,46}$. Another limitation is the inability of this study to account for diagnostic delays, which are common in both ED and PsA. In both cases, for a retrospective study to detect a case, the patient must experience the symptoms significantly enough to prompt the patient to seek medical attention. In the case of PsA, some men might have a higher tolerance for pain, or subscribe to a culture that is less likely to present with pain as a chief complaint. This means that patients will present at various stages of psoriatic disease, with some presenting with mild early disease and others presenting with more severe disease at diagnosis. Studies, including a previous metaanalysis, have shown that approximately $10-15 \%$ of patients with psoriasis actually have symptoms and meet diagnostic criteria for PsA, despite not being previously diagnosed ${ }^{48,49}$. Another study found that, in 2000, the time from initial symptom onset to diagnosis of PsA was approximately 53 months, but this lag in diagnosis has decreased in 2011 to approximately $3-4$ months $^{50}$. Given this range in time to diagnosis, our cohort, which was initially diagnosed between 1970 and 2008, may be particularly heterogeneous in their functional status and disease severity at time of diagnosis. Similarly, some men may seek treatment early for mild $\mathrm{ED}$, whereas others may never seek treatment due to a variety of reasons, including being sexually inactive or having medical conditions that make sexual activity unsafe ${ }^{45}$. Future studies could circumvent this complication by utilizing a prospective design with more quantitative testing. In addition, we examined some of the known confounders of ED and the primary treatment strategies. However, we did not examine other baseline traditional risk factors for ED such as smoking, obesity, metabolic syndrome, or depression, which are higher in PsA compared to the general population. Other limitations include the retrospective nature of the current study with all the respective limitations, and the predominantly White population of Olmsted County, possibly limiting generalizability to more diverse populations.

This study showed an increased incidence of ED in men with PsA in relation to a comparator cohort; however, it did not reach statistical significance. Future studies in larger population-based areas could further inform this observation and confirm if ED is more prevalent in men with PsA. In addition, future studies could determine if the risk of ED in PsA is driven by traditional $\mathrm{CV}$ risk factors, as in the general population, or driven by psoriatic inflammation. In addition, it has been shown that psoriatic disease activity is associated with increased risk of sexual dysfunction in men with psoriasis ${ }^{14,15,16}$, so it is possible that a PsA cohort with well-controlled disease activity might have a lower rate of ED. The potential effect of intensification of PsA treatment with better disease control on ED could also be informed by future studies. ED has been used as a marker of men with subclinical CVD in the general population, guiding CV intervention. It is currently unclear whether this is true for men with PsA. Future studies, which may be able to ascertain the incidence of ED in larger, perhaps country-based populations of PsA, could help to more clearly define both the risk of ED and its association with $\mathrm{CV}$ risk and, perhaps, predictive ability. The prospective use of ED screening, including penile tumescence studies, use of the International Index of Erectile Function questionnaires, and ultrasound-based diagnostics of atherosclerosis could all help to further determine if there is a predictive value of early ED pathogenesis to the development of CVD. Collaborations between experts in rheumatology and andrology/urology could optimize these studies in order to best inform both fields. Given the relevance of ED to CVD, more studies on ED risk could inform both the management of sexual dysfunction and CV risk in men with PsA. In addition, clinicians caring for patients with PsA should be aware of the possibility that men with PsA may be at higher risk of ED, and they should screen their patients accordingly to increase quality of life, and perhaps eventually, to help ascertain future $\mathrm{CV}$ risk.

\section{REFERENCES}

1. Ernste FC, Sanchez-Menendez M, Wilton KM, Crowson CS, Matteson EL, Maradit Kremers H. Cardiovascular risk profile at the onset of psoriatic arthritis: a population-based cohort study. Arthritis Care Res 2015;67:1015-21.

2. Jamnitski A, Symmons D, Peters MJ, Sattar N, McInnes I, Nurmohamed MT. Cardiovascular comorbidities in patients with psoriatic arthritis: a systematic review. Ann Rheum Dis 2013;72:211-6.

3. Kondratiouk S, Udaltsova N, Klatsky AL. Associations of psoriatic arthritis and cardiovascular conditions in a large population. The Perm J 2008;12:4-8.

4. Li L, Hagberg KW, Peng M, Shah K, Paris M, Jick S. Rates of cardiovascular disease and major adverse cardiovascular events in patients with psoriatic arthritis compared to patients without psoriatic arthritis. J Clin Rheumatol 2015;21:405-10.

5. Polachek A, Touma Z, Anderson M, Eder L. Risk of cardiovascular morbidity in patients with psoriatic arthritis: a meta-analysis of observational studies. Arthritis Care Res 2017;69:67-74.

6. Popescu C, Pintilie AM, Bojinca V, Balanescu A, Ionescu R. Cardiovascular risk in psoriatic arthritis - a cross-sectional study. Maedica 2014;9:19-24.

7. Sinnathurai P, Buchbinder R, Hill C, Lassere M, March L. Comorbidity in psoriatic arthritis and rheumatoid arthritis. Intern Med J 2018;48:1360-8.

8. Tam LS, Tomlinson B, Chu TT, Li M, Leung YY, Kwok LW, et al. Cardiovascular risk profile of patients with psoriatic arthritis compared to controls - the role of inflammation. Rheumatology 2008;47:718-23.

9. Molina-Leyva A, Salvador-Rodriguez L, Martinez-Lopez A, Ruiz-Carrascosa JC, Arias-Santiago S. Association between psoriasis and sexual and erectile dysfunction in epidemiologic studies: a systematic review. JAMA Dermatol 2019;155:98-106.

10. Chen YJ, Chen CC, Lin MW, Chen TJ, Li CY, Hwang CY, et al. Increased risk of sexual dysfunction in male patients with psoriasis: a nationwide population-based follow-up study. J Sex Med 2013;10:1212-8.

11. Gupta MA, Gupta AK. Psoriasis and sex: a study of moderately to severely affected patients. Int J Dermatol 1997;36:259-62.

12. Sampogna F, Gisondi P, Tabolli S, Abeni D; IDI Multipurpose Psoriasis Research on Vital Experiences investigators. Impairment of sexual life in patients with psoriasis. Dermatology 2007;214:144-50.

13. Gladman DD. Mortality in psoriatic arthritis. Clin Exp Rheumatol 2008;5 Suppl 51:S62-5.

14. Gladman DD, Ang M, Su L, Tom BD, Schentag CT, Farewell VT. Cardiovascular morbidity in psoriatic arthritis. Ann Rheum Dis 2009;68:1131-5.

15. Zhu TY, Li EK, Tam LS. Cardiovascular risk in patients with psoriatic arthritis. Int J Rheumatol 2012;2012:714321. 
16. Egeberg A, Skov L, Hansen PR, Gislason GH, Wu JJ, Thyssen JP, et al. Duration of psoriatic arthritis as a risk factor for myocardial infarction. Rheumatol Adv Pract 2018;2:rky011.

17. Cooksey R, Brophy S, Kennedy J, Gutierrez FF, Pickles T, Davies $\mathrm{R}$, et al. Cardiovascular risk factors predicting cardiac events are different in patients with rheumatoid arthritis, psoriatic arthritis, and psoriasis. Semin Arthritis Rheum 2018;48:367-73.

18. Liew JW, Ramiro S, Gensler LS. Cardiovascular morbidity and mortality in ankylosing spondylitis and psoriatic arthritis. Best Pract Res Clin Rheumatol 2018;32:369-89.

19. Calmasini FB, Klee N, Webb RC, Priviero F. Impact of immune system activation and vascular impairment on male and female sexual dysfunction. Sex Med Rev 2019;7:604-13.

20. Lotti F, Maggi M. Sexual dysfunction and male infertility. Nat Rev Urol 2018;15:287-307.

21. Mitidieri E, Cirino G, d'Emmanuele di Villa Bianca R, Sorrentino R. Pharmacology and perspectives in erectile dysfunction in man. Pharmacol Ther 2020;208:107493.

22. Yafi FA, Jenkins L, Albersen M, Corona G, Isidori AM, Goldfarb S, et al. Erectile dysfunction. Nat Rev Dis Primers 2016;2:16003.

23. Caretta N, De Rocco Ponce M, Minicuci N, Palego P, Valente U, Garolla A, et al. Penile Doppler ultrasound predicts cardiovascular events in men with erectile dysfunction. Andrology 2019;7:82-7.

24. Ioakeimidis N, Vlachopoulos C, Rokkas K, Kratiras Z, Angelis A, Samentzas A, et al. Dynamic penile peak systolic velocity predicts major adverse cardiovascular events in hypertensive patients with erectile dysfunction. J Hypertens 2016;34:860-8.

25. Chung E. Contemporary and novel imaging studies for the evaluation of erectile dysfunction. Med Sci 2019;7:87.

26. Zou Z, Lin H, Zhang Y, Wang R. The role of nocturnal penile tumescence and rigidity (NPTR) monitoring in the diagnosis of psychogenic erectile dysfunction: a review. Sex Med Rev 2019;7:442-54.

27. Montorsi P, Ravagnani PM, Galli S, Rotatori F, Briganti A, Salonia A, et al. Common grounds for erectile dysfunction and coronary artery disease. Curr Opin Urol 2004;14:361-5.

28. Montorsi P, Ravagnani PM, Galli S, Rotatori F, Briganti A, Salonia A, et al. The artery size hypothesis: a macrovascular link between erectile dysfunction and coronary artery disease. Am J Cardiol 2005;96:19M-23M.

29. Kapur V, Schwarz ER. The relationship between erectile dysfunction and cardiovascular disease. Part I: pathophysiology and mechanisms. Rev Cardiovasc Med 2007;8:214-9.

30. Shridharani AN, Brant WO. The treatment of erectile dysfunction in patients with neurogenic disease. Transl Androl Urol 2016; 5:88-101.

31. Del Popolo G, Cito G, Gemma L, Natali A. Neurogenic sexual dysfunction treatment: a systematic review. Eur Urol Focus 2020;6:868-76

32. Bodie JA, Beeman WW, Monga M. Psychogenic erectile dysfunction. The Int J Psychiatry Med 2003;33:273-93.

33. Montorsi P, Ravagnani PM, Galli S, Rotatori F, Veglia F, Briganti A, et al. Association between erectile dysfunction and coronary artery disease. Role of coronary clinical presentation and extent of coronary vessels involvement: the COBRA trial. Eur Heart J 2006;27:2632-9.

34. Baumhäkel M, Böhm M. Erectile dysfunction correlates with left ventricular function and precedes cardiovascular events in cardiovascular high-risk patients. Int J Clin Pract 2007;61:361-6.

35. Zhao SS, Miller N, Harrison N, Duffield SJ, Dey M, Goodson NJ. Systematic review of mental health comorbidities in psoriatic arthritis. Clin Rheumatol 2020;39:217-25.
36. El-Mawla A, Hashim AA, Zidan AS. Assessment of the effect of psoriasis on male sexual function. Human Androl 2018;8:69-75.

37. Tasliyurt T, Bilir Y, Sahin S, Seckin HY, Kaya SU, Sivgin H, et al. Erectile dysfunction in patients with psoriasis: Potential impact of the metabolic syndrome. Eur Rev Med Pharmacol Sci 2014; 18:581-6.

38. Bardazzi F, Odorici G, Ferrara F, Magnano M, Balestri R, Patrizi A. Sex and the PASI: patients affected by a mild form of psoriasis are more predisposed to have a more severe form of erectile dysfunction. J Eur Acad Dermatol Venereol 2016;30:1342-8.

39. Perez-Garcia LF, Te Winkel B, Carrizales JP, Bramer W, Vorstenbosch S, van Puijenbroek E, et al. Sexual function and reproduction can be impaired in men with rheumatic diseases: a systematic review. Semin Arthritis Rheum 2020;50:557-73.

40. St Sauver JL, Grossardt BR, Leibson CL, Yawn BP, Melton LJ 3rd, Rocca WA. Generalizability of epidemiological findings and public health decisions: An illustration from the Rochester Epidemiology Project. Mayo Clin Proc 2012;87:151-60.

41. Wilton KM, Crowson CS, Matteson EL. Malignancy incidence in patients with psoriatic arthritis: a comparison cohort-based incidence study. Clin Rheumatol 2016;35:2603-7.

42. Taylor W, Gladman D, Helliwell P, Marchesoni A, Mease P, Mielants $\mathrm{H}$, et al; CASPAR Study Group. Classification criteria for psoriatic arthritis: development of new criteria from a large international study. Arthritis Rheum 2006;54:2665-73.

43. Gooley TA, Leisenring W, Crowley J, Storer BE. Estimation of failure probabilities in the presence of competing risks: new representations of old estimators. Stat Med 1999;18:695-706.

44. Inman BA, Sauver JLS, Jacobson DJ, McGree ME, Nehra A, Lieber MM, et al., editors. A population-based, longitudinal study of erectile dysfunction and future coronary artery disease. Mayo Clinic Proc 2009;84:108-13.

45. Gades NM, Jacobson DJ, McGree ME, St Sauver JL, Lieber MM, Nehra A, et al. Longitudinal evaluation of sexual function in a male cohort: the Olmsted county study of urinary symptoms and health status among men. J Sex Med 2009;6:2455-66.

46. Molina-Leyva A, Almodovar-Real A, Carrascosa JC, Molina-Leyva I, Naranjo-Sintes R, Jimenez-Moleon JJ. Distribution pattern of psoriasis, anxiety and depression as possible causes of sexual dysfunction in patients with moderate to severe psoriasis. An Bras Dermatol 2015;90:338-45.

47. Molina-Leyva A, Molina-Leyva I, Almodovar-Real A, Ruiz-Carrascosa JC, Naranjo-Sintes R, Jimenez-Moleon JJ. Prevalence and associated factors of erectile dysfunction in patients with moderate to severe psoriasis and healthy population: a comparative study considering physical and psychological factors. Arch Sex Behav 2016;45:2047-55.

48. Villani AP, Rouzaud M, Sevrain M, Barnetche T, Paul C, Richard MA, et al. Prevalence of undiagnosed psoriatic arthritis among psoriasis patients: systematic review and meta-analysis. J Am Acad Dermatol 2015;73:242-8.

49. Coates LC, Savage L, Waxman R, Moverley AR, Worthington S, Helliwell PS. Comparison of screening questionnaires to identify psoriatic arthritis in a primary-care population: a cross-sectional study. Br J Dermatol 2016;175:542-8.

50. Sørensen J, Hetland ML; all departments of rheumatology in Denmark. Diagnostic delay in patients with rheumatoid arthritis, psoriatic arthritis and ankylosing spondylitis: results from the Danish nationwide DANBIO registry. Ann Rheum Dis 2015;74:e12. 